\title{
Structural Analysis of T - Beam Based on ANSYS Shuai $\mathrm{Li}^{1}$ and Yuanyuan Cai ${ }^{2}$
}

${ }^{1}$ College of Mechanical and Electrical Engineering, Zaozhuang University Shandong province, 277160;

\section{${ }^{2}$ Zaozhuang Experimental School, Zaozhuang, Shandong 277100}

\begin{abstract}
The typical characteristic of modern industry is the great number of computer applications, no matter product development, design, or analysis, manufacturing process, the computer application has greatly improved the efficiency and quality. Computer-aided engineering (CAE) is one of the essential links, it is the perfect combination of computer technology and modern engineering methods. In the actual production process, we will meet a variety of mechanical structure analysis problems: such as mechanical structure of the force, deformation and internal stress, etc. Structural analysis is one of the most commonly used applications in finite element method. This paper briefly describes the seven types of structural analysis of ANSYS. Then, combine the analysis of equivalent stress field and strain field distribution of T-beam, introduce the general steps of ANSYS structural analysis.
\end{abstract}

Keyword: CAE; Structural analysis; T - Beam; Stress field; Strain field

\section{基于 ANSYS 的 $\mathbf{T}$ 型梁结构分析}

\section{李帅 ${ }^{1}$ 蔡媛媛 ${ }^{2}$}

(1. 本庄学院机电工程学院, 山东 本庄 $277160 ； 2$. 本庄市实验学校, 山东 本庄 277100）

摘要: 现代工业的典型特征是计算机的大量应用, 无论是产品开发、设计, 还是分析、制造过程中, 计算机的应用都极 大地提高了效率和质量。计算机辅助工程 (CAE) 就是其中必不可少的一个环节, 它是计算机技术和现代工程方法的完美结 合。在实际生产过程中, 我们会遇到各种各样的机械结构分析问题: 如机械结构受力, 变形及内部应力情况等等。结构分析 是有限元方法中最常用的一个应用领域, 本文简要叙述了 ANSYS 的七种结构分析类型, 然后结合 T 型梁的等效应力场和应 变场分布的分析, 介绍了 ANSYS 结构分析的一般步骤。

关键词: CAE; 结构分析; $\mathrm{T}$ 型梁; 应力场; 应变场

中图分类号: TP391.9 文献标志码：A

引言

结构分析是有限元分析方法最常用的一个应用领域, 结构是一个广义的概念, 包括土木工程结构, 如 桥梁和建筑物; 汽车结构, 如车身骨架; 航空结构, 如飞机机身等; 同时, 还包括机械零部件, 如活塞、 传动器等等。结构分析中计算得出的基本未知量 (节点自由度) 是位移, 其他的一些未知量, 如应变、应 力和反作用力等, 均可通过节点位导出。利用 ANSYS 软件对机械模型进行仿真模拟计算, 通过应力、应 变云图直观展示构件的性能特点, 从而为解决机械结构中常见的问题提供理论依据 ${ }^{[1-3]}$ 。ANSYS 仿真分析 的结果可以帮助设计人员对实际生产方案作出准确的判断, 节省物力财力, 为提高生产效率及缩短设计研 发周期有很大的作用 ${ }^{[4]}$ 。本文结合典型工程实例介绍了 ANSYS 结构分析的基本方法和分析过程。

\section{1、ANSYS 结构分析类型}

ANSYS (Analysis System) 是一个集结构、热、流体、电磁和声学分析为一体的大型通用有限元软件, 可广泛用于石油化工、航空航天、机械制造、能源、汽车等工业及科学研究领域 ${ }^{[5]}$ 。ANSYS 的结构分析主 
要包括以下七种类型:

（1）结构静力分析。用来计算在固定不变的载荷作用下结构的效应，即由于稳态外载引起的系统部 件的位移、应力、应变及力。同时, 结构静力分析还可以计算那些固定不变的惯性载荷以及那些可以近似 等价为静力作用的随时变化的载荷对结构的影响。

（2）模态分析。用于确定设计中的结构或机器部件的振动特性（固有频率）和振型，也是瞬态动力 学分析、谐响应分析和谱分析的起点。

（3）谐波分析。用于分析持续的周期载荷在结构系统中产生的持续的周期响应（谐响应），以及确定 线性结构承受随时间按正弦（简谐）规律变化的载荷时稳定响应的一种技术。

（4）瞬态动力学分析。用于确定承受任意的随时间变化载荷的结构动力学响应的一种方法。可以用 瞬态动力学确定结构在静载荷、瞬态载荷和简谐载荷的随意组合作用下随时间变化的位移、应变、应力及 力。

（5）谱分析。它是模态分析的应用拓广，用于计算由于响应或 PSD 输入（随时振动）引起的应力和 应变。

（6）结构屈曲分析。它是一种用于确定结构的屈曲载荷（使结构变得不稳定的临界载荷）和屈曲模 态（结构屈曲响应的特征形态）的技术。

（7）显式动力学分析。用于计算高度非线性动力学和复杂的接触问题。

\section{2、实例导入}

下面结合具体实例介绍 ANSYS 结构仿真分析的过程，问题描述： $\mathrm{T}$ 型梁的结构如图 1 所示。现将其 一端固定, 在另一端上端面中心施加 $5000 \mathrm{~N}$ 的集中力 $\mathrm{P}$, 求此时 $\mathrm{T}$ 型梁内部应力、应变场分布及挠度。该 $\mathrm{T}$ 型梁材料的弹性模量为 $120 \mathrm{GPa}$, 泊松比为 0.26 , 梁的跨度为 $1000 \mathrm{~mm}$ 。
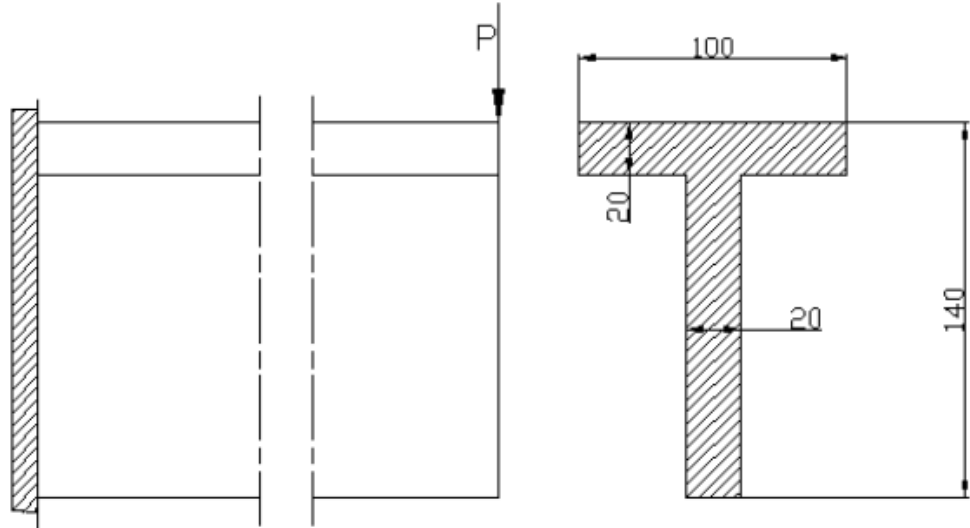

图 1 T 型梁结构示意图

\section{3、 $\mathbf{T}$ 型梁的结构分析}

\section{1 建立工作文件名和工作标题}

虽然该项工作不做强制要求，但是在对多个工程问题进行分析时推荐使用工作文件名和工作标题。文 件名是用来识别 ANSYS 作业的, 通过为分析的工程指定文件名, 可以确保文件不被覆盖。如果用户在分 析开始时没有定义工作文件名，则所有文件名都被默认地设置为 file。工作标题用来标识当前分析的某种 信息，用于提示如分析对像，分析工况，分析性质等 ${ }^{[6]}$ 。 


\section{Command: /TITLE, A FORCE TO THE BEAM}

\section{2 创建几何模型}

ANSYS 提供两种生成模型的方法: 用 ANSYS 直接创建实体模型和输入计算机辅助设计系统创建的模 型。采用实体建模有自底向上建模和自顶向下建模两种方法; 所谓自底向下建模是指先定义关健点, 然后 利用关健点定义较高级的实体图元 (即线、图和体); 而自顶向下建模是指生成体素, 属于该体素的较低 级图元会由 ANSYS 自动生成 ${ }^{[7]}$ 。在实体建模时这两种建模技术可以自由结合。

Command: RECTNG, 0, 0.1, 0, 0.02

RECTNG, 0.04, 0.06, -0.12, 0

AADD $, 1,2$

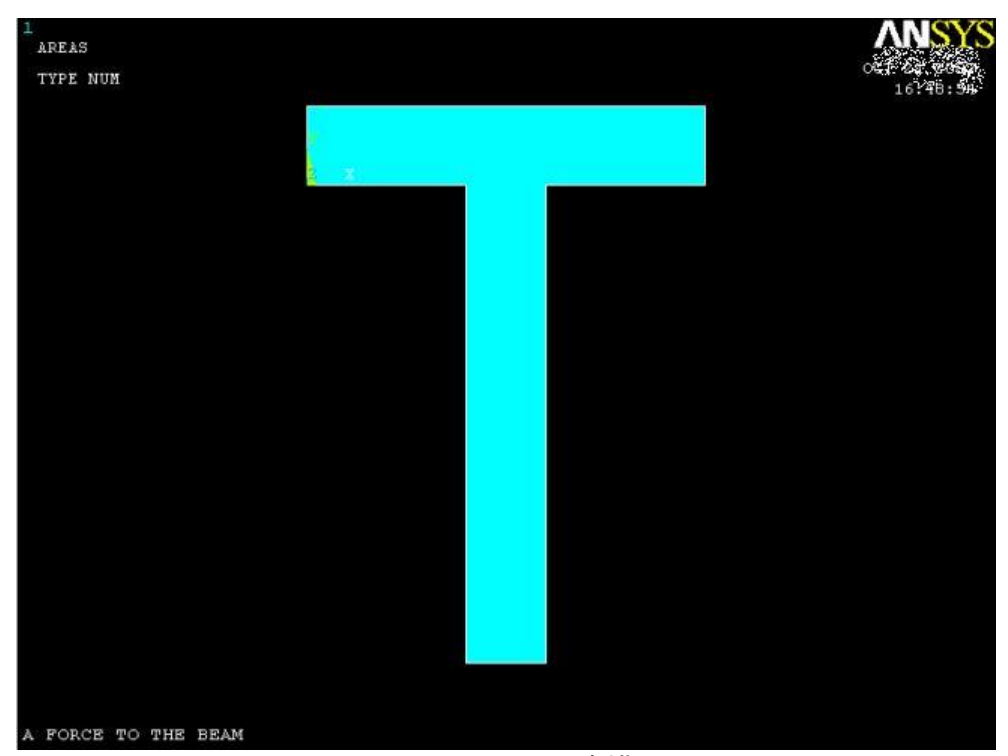

图 2 ANSYS 建模

利用 ANSYS 的 CAD 系统接口, 将用户熟悉的 UG, PRO/E 等外部软件建立好的模型, 导入到 ANSYS 中进行分析, 从而避免了重复创建现有的 CAD 模型, 如图 2 所示。

\section{3 定义单元类型}

ANSYS 提供了近 200 种不同的单元类型, 每一种单元类型都有自己特定的编号和单元类型名, 如 PLANE182、SOLID90、SHELL208 等; 单元关键字定义了单元的不同特性, 如轴对称、平面应力等, 用 户需根据需要选择相应的单元类型, 并设置关键字。

Command: ET, 1, PLANE82

ET, 2, SOLID185

\section{4 定义材料性能参数}

在所有的分析中都要输入材料属性, 材料属性根据分析问题的物理环境不同而不同。如在结构分析中 必须输入材料的弹性模量、泊松比; 在热结构耦合分析中必须输入材料的热导、线膨胀系数; 如果在分析 过程中需要考虑重力、惯性力, 则必须要输入材料的密度 ${ }^{[8]}$ 。

Command: MP, EX , 1, 1.2E11

MP, PRXY, 1, 0.26

ANSYS 定义了 100 多种材料模型, 用户只需要按照模型格式输入相关数据即可定义常用材料和某些 特定材料的属性。除了在磁场分析之外, 在输入数据时用户不需要指定 ANSYS 所用的单位, 但要注意确 保所有输入量的单位必须保持一致。

\section{5 划分网格}


ANSYS 提供两种网格划分方法: 自由网格和映射网格。自由网格体现在划分网格没有特定准则，对 单元形状无限制, 生成的单元不规则, 基本适用于所有的模型。映射网格则要求满足一定的规则, 且映射 面网格只包含四边形或三角单元，而映射体网格只包含六面体单元 ${ }^{[9]}$ 。 $\mathrm{T}$ 型梁的网格划分如图 3 所示。

Command: LSEL, S, , , 1, 3, 2

LESIZE, ALL, , , 2

ALLSEL

LESIZE, $2,,, 10$

LSEL, S, , , 7, 8

LESIZE, ALL, , , 4

ALLSEL

LESIZE, 4, , , 2

LSEL, S , , , 5, 6

LESIZE, ALL, , , , 10

ALLSEL

AMESH, 1

ESIZE, 60

VOFFST, 1, 1

\section{6 加载求解}

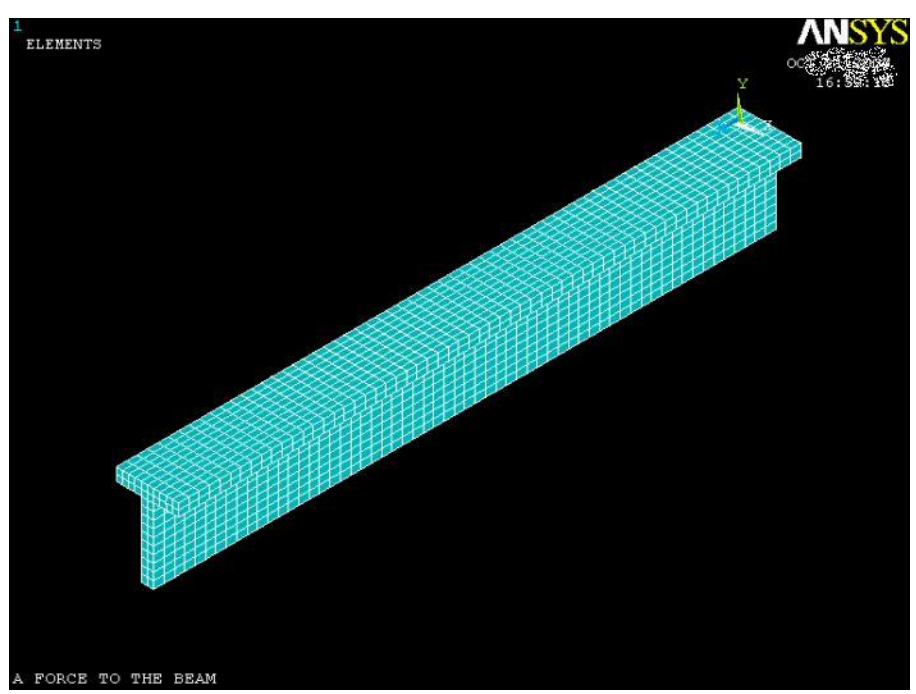

图 3 网格划分

在有限元模型建立之后, 可以运用 SOLUTION 处理器定义分析类型和分析选项, 施加载荷, 指定载 荷步长, 进行求解。

Command: ANTYPE, STATIC

NSEL, S, LOC, Z

$\mathrm{D}, \mathrm{ALL}, \mathrm{UX}$

$\mathrm{D}, \mathrm{ALL}, \mathrm{UY}$

$\mathrm{D}, \mathrm{ALL}, \mathrm{UZ}$

\section{ALLSEL}

F, 710, FY, -5000

ALLSEL 


\section{SOLVE}

\section{7 查看结果}

程序计算完成之后, 可以通过通用后处理器 POST1 和时间历程后处理器POST26 查看求解结果。POST1 用于查看整个模型或部分模型在某一时间步的计算结果, POST26 后处理器用于查看模型的特点在所有时 间步内的计算结果。等效应力场和应变场分布见图 4、5。

Command: PLNSOL, U, Y

$$
\begin{aligned}
& \text { PLNSOL, S, X } \\
& \text { PLNSOL, S, Y, } \\
& \text { PLNSOL, S, Z } \\
& \text { PLNSOL, S, EQV } \\
& \text { PLNSOL, EPTO, EQV }
\end{aligned}
$$

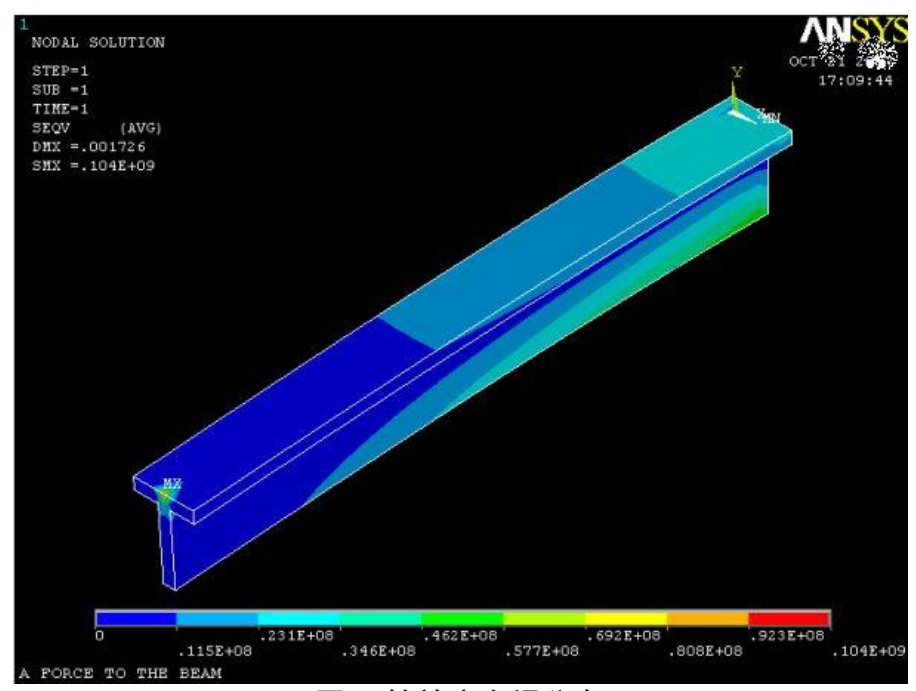

图 4 等效应力场分布

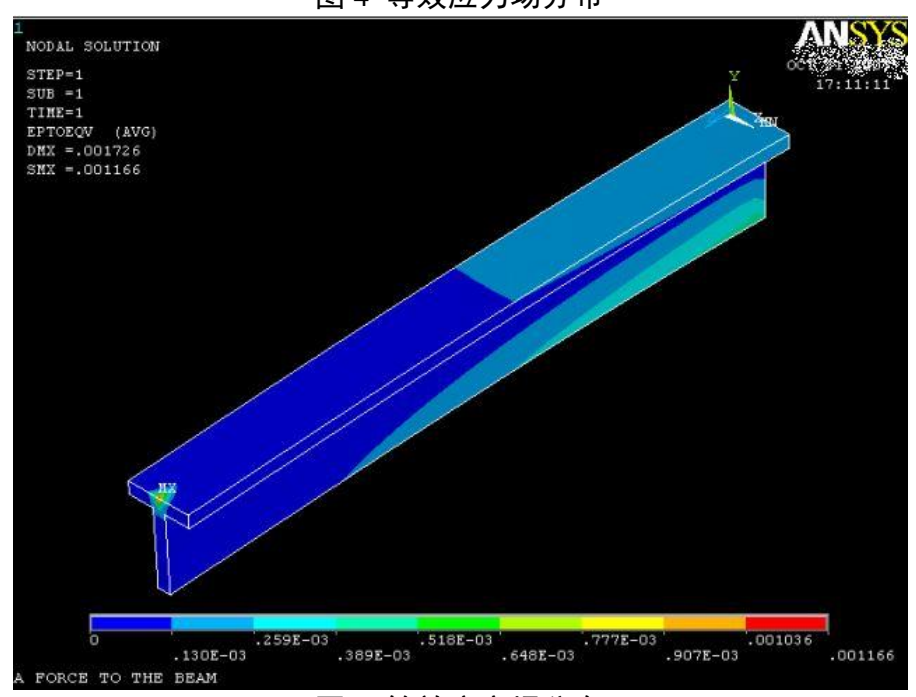

图 5 等效应变场分布

\section{4、ANSYS 在结构分析中的发展趋势}

由以上实例可以看出, ANSYS 在机械结构分析中的直观性和准确性较高, 不同颜色区域的应力场和 应变场分布有助于工程设计人员快速解决复杂的问题, 避免机械部件的局部受力过大而失效。随着计算机 技术的发展, ANSYS 提供的机械工程仿真技术将越来越成熟、完善, 设计人员以真正耦合的方式使用 ANSYS 技术, 可获得符合现实条件的解决方案 ${ }^{[10]}$ 。ANSYS 软件可让用户更深入的分析研究, 从而解决更 
多种类的问题，处理更为复杂的情况。

因此, ANSYS 产品以其灵活的仿真性能将会被越来越广泛的关注和应用。

\section{参考文献:}

[1] 王亚利。ANSYS 软件在机械结构分析中的应用 [J]。价值工程，2014（3)，203-204。

[2] 谷俊斌, 贾宏玉。ANSYS 软件在工程力学专业教学中的应用 [J]。中国冶金教育, 2013 （4），45-46。

[3] 苏荣华, 梁冰。结构仿真分析-ANSYS 应用 [M]。沈阳：东北大学出版社, 2015。

[4] 张胜民。基于有限元软件 ANSYS7. 0 的结构分析 [M]。北京：清华大学出版社，2013。

[5] 张朝晖。ANSYS 8.0 结构分析及实例解析 [M]。北京: 清华大学出版社, 2005。

[6] 黄一江。ANSYS 结构优化设计在机械设计中的应用 [J]。应用技术，2013（4），23-25。

[7] 刘伟, 高维成等。ANSYS12.0 宝典 [M]。北京：电子工业出版社, 2015。

[8］赵晓光，魏志卿。基于 ANSYS 的结构可靠性分析 [J]。国外建材科技，2008（5)，94-96。

[9] 王呼佳, 陈洪军。ANSYS 工程分析进阶实例 [M]。北京: 中国水利水电出版社, 2016。

[10] 邓凡平。ANSYS12.0 有限元分析自学手册 [M]。北京：人民邮电出版社，2014。

\section{References:}

[1] Wang Yali. The Application of ANSYS Software in Mechanical Structure Analysis. [J]. Value Engineering, 2014 (3), 203-204.

[2] Gu Junbin, Jia Hongyu. The Application of ANSYS Software in the Teaching of Engineering Mechanics. [J]. China Metallurgical Education, 2013 (4), 45-46.

[3] Su Ronghua, Liangbing. Structural Simulation Analysis - ANSYS Application [M]. Shenyang: Northeastern University Press, 2015.

[4] Zhang Shengmin Structural Analysis Based on Finite Element Software ANSYS7.0 [M]. Beijing: Tsinghua University Press, 2013

[5] Zhang Chaohui Structural Analysis and Case Analysis of ANSYS8.0 [M]. Beijing: Tsinghua University Press, 2005

[6] Huang Yijiang Application of ANSYS Structure Optimization Design in Mechanical Design [J]. Applied Technology, 2013 (4), 23-25.

[7] Liu Wei Gao Weicheng, etc. [M] ANSYS12.0 Collection Beijing: Electronic Industry Press, 2015 .

[8] Zhao Xiaoguang, Wei Zhiqing Structural Reliability Analysis Based on ANSYS [J]. Foreign Building Materials Technology, 2008 (5), 94-96.

[9] Wang Hujia, Chen Hongjun Further Examples of ANSYS Engineering Analysis [M]. Beijing: China Water Resources and Hydropower Press, 2016.

[10]Deng Fanping ANSYS12.0 Self - Study Manual of Finite Element Analysis [M]. Beijing: People's Posts and Telecommunications Press, 2014. 